\title{
Repositório Colaborativo para apoiar a adoção de Metodologias Ativas no Ensino de Programação
}

\author{
Ivanilse Calderon Ribeiro \\ Instituto de Computação (IComp) \\ Universidade Federal do Amazonas \\ Manaus, AM - Brasil \\ ivanilse.calderon@icomp.ufam.edu.br
}

\author{
Williamson Silva \\ Departamento de Ciência da \\ Computação \\ Universidade Estadual do Paraná \\ Apucarana, PR - Brasil \\ williamson.silva@gmail.com
}

\author{
Eduardo Luzeiro Feitosa \\ Instituto de Computação (IComp) \\ Universidade Federal do Amazonas \\ Manaus, AM -Brasil \\ efeitosa@icomp.ufam.edu.br
}

O ensino de programação é um processo complexo [1], pois requer que os estudantes desenvolvam ao longo da aprendizagem diferentes habilidades, tais como capacidade de abstração, resolução de problemas, raciocínio e pensamento lógico [2-4]. Tradicionalmente, o ensino de programação se dá por meio de aulas expositivas combinadas com exercícios que descrevem problemas ao qual os estudantes devem solucionar [2,5-6]. Essa forma de ensino vem recebendo diversas críticas, uma vez que a transmissão do conhecimento é realizada de forma passiva [7-9]. Visando minimizar este problema, docentes tentam adaptar ou empregar novas estratégias de ensino para proporcionar um ambiente de aprendizagem desafiador e engajador para os estudantes $[5,10]$.

Neste sentido, o uso de Metodologias Ativas (MAs) vêm ganhando destaque entre os docentes [11-12]. De acordo com Koening [13], as MAs baseiam-se na teoria Construtivista, em que a aprendizagem é responsabilidade do estudante. As MAs criam situações de aprendizagem para que os estudantes construam conhecimentos sobre os conteúdos aprendidos, desenvolvendo a capacidade crítica e a reflexão sobre as práticas que realizam, bem como explorando atitudes, valores pessoais e aprendam-fazendo (learning by doing) $[12,14,15]$.

Apesar das evidências positivas em relação às MAs, a adoção por parte dos docentes ainda é relativamente baixa [16-18]. Isso vem ocorrendo devido às diversas barreiras que os professores enfrentam na adoção das MAs, tais como: (a) falta de tempo para o planejamento das aulas adotando MAs [16-17]; (b) dificuldade de cumprir todo o conteúdo da disciplina [16,19]; (c) rejeição por parte dos estudantes em relação à utilização de novas metodologias de ensino; (d) falta de informação sobre como implementar as MAs nas aulas $[3,19]$.

Dado o contexto apresentado, esta pesquisa tem como objetivo apoiar a adoção das MAs para o ensino de programação, minimizando as barreiras e/ou desafios enfrentados pelos docentes. Esta pesquisa está sendo guiada pela metodologia de Design Science Research (DSR) [20-21] para delimitar o problema de pesquisa, o desenvolvimento, a avaliação e evolução do artefato. A

Fica permitido ao(s) autor(es) ou a terceiros a reprodução ou distribuição, em parte ou no todo, do material extraído dessa obra, de forma verbatim, adaptada ou remixada, bem como a criação ou produção a partir do conteúdo dessa obra, para fins não comerciais, desde que sejam atribuídos os devidos créditos à criação original, sob os termos da licença CC BY-NC 4.0.

EduComp'21, Abril 26-30, 2021, Jataí, Goiás, Brasil (On-line)

(C)2021 Copyright mantido pelo(s) autor(es). Direitos de publicação licenciados à

Sociedade Brasileira de Computação (SBC). proposta inicial é desenvolver um repositório colaborativo aberto em que os docentes possam identificar, selecionar, adotar, discutir, comentar, avaliar e possivelmente colaborar com (novas ou não) MAs utilizadas durante o ensino de programação.

O repositório auxiliará o docente na identificação e escolha de MA(s) de acordo com o seu contexto de ensino e que atenda às suas necessidades pedagógicas. O repositório também disponibilizará um conjunto de guidelines que contará com o passo a passo para guiar os docentes durante a adoção das MAs. Desta forma, os docentes não precisarão buscar, em vários artigos científicos ou livros, formas de como conduzir uma determinada MA em sala de aula. Com a elaboração do repositório, estas informações ficarão disponíveis em apenas um único lugar. Além disso, em razão da proposição de novas MAs, o repositório será colaborativo e aberto à comunidade acadêmica. Assim, docentes poderão contribuir com MAs adotadas, avaliando positivamente ou não o uso de uma determinada MA. Esta avaliação permitirá que os demais docentes possam compartilhar com a comunidade docente suas experiências de uso de uma MA. Isso ajudará os demais docentes durante o processo de adoção ou não de uma determinada MA. É importante mencionar que o repositório está na fase de ideação, ou seja, ainda está sendo realizada a identificação e o mapeamento das MAs, materiais de apoio, informações e artefatos que poderão ajudar os professores durante a adoção de uma MA. A coleta e curadoria destas informações apoiarão na concepção e no desenvolvimento do artefato proposto (repositório colaborativo aberto).

Por fim, para avaliar a viabilidade de uso e evoluir o repositório, pretende-se conduzir estudos experimentais quantitativos (questionários - Modelo de Aceitação de Tecnologia, surveys) e qualitativos (estudos de caso, entrevistas e sessões de grupo focal) com docentes que ministram disciplinas de programação. Espera-se que, a partir do uso do repositório, algumas barreiras enfrentadas pelos docentes durante a adoção de MAs sejam minimizadas, uma vez que a literatura confirma que docentes e pesquisadores em Educação estão obtendo resultados significativamente melhores ao experimentar novas intervenções e abordagens pedagógicas durante $\mathrm{o}$ processo de ensinoaprendizagem [22].

\section{AGRADECIMENTOS}

Os autores agradecem ao apoio financeiro fornecido de forma indireta das seguintes instituições de ensino: Universidade Federal do Amazonas (UFAM), Instituto Federal de Educação, Ciência e 
Tecnologia de Rondônia (IFRO) / Campus Porto Velho Zona Norte e da Universidade Estadual do Paraná (UNESPAR) / Campus Apucarana.

\section{REFERÊNCIAS}

[1] Andrew Luxton-Reilly, Ibrahim Albluwi, Brett A. Becker, Michail Giannakos, Amruth N. Kumar, Linda Ott, James Paterson, Michael James Scott, Judy Sheard, and Claudia Szabo. 2018. Introductory programming: a systematic literature review. In Proceedings Companion of the 23rd Annual ACM Conference on Innovation and Technology in Computer Science Education, pp. 55-106.

[2] Adalbert Gerald Soosai Raj, Jignesh Patel, and Richard Halverson. 2018. Is More Active Always Better for Teaching Introductory Programming? In2018 International Conference on Learning and Teaching in Computing and Engineering (LaTICE), IEEE, 103-109.

[3] Maria Ivanilse Calderon Ribeiro, and Odette Mestrinho Passos. 2020. A Study on the Active Methodologies Applied to Teaching and Learning Process in the Computing Area. In IEEE Access, (2020), 219083-219097.

[4] Chandra Turpen, Melissa Dancy, and Charles Henderson. 2016. Perceived affordances and constraints regarding instructors' use of Peer Instruction: Implications for promoting instructional change. In Physical Review Physics Education Research 12, 1 (2016), 010116.

[5] Essi Lahtinen, Kirsti Ala-Mutka, and Hannu-Matti Järvinen. 2005. A study of the difficulties of novice programmers. In ACM SIGCSE Bulletin 37, 3 (2005), 14 18.

[6] Diego Teixeira Witt, and Avanilde Kemczinski. 2020. Metodologias de Aprendizagem Ativa Aplicadas à Computação: Uma Revisão da Literatura. In Informática na educação: teoria \& prática 23, 1 (2020).

[7] Lilian Bacich and José Moran. Metodologias Ativas para uma Educação Inovadora: Uma Abordagem Teórico-Prática. Penso Editora.

[8] Chicon P. M., C. R. Quaresma, and S. B. Garcês. 2019. Aplicação do Método de ensino Peer Instruction para o Ensino de Lógica de Programação com acadêmicos do Curso de Ciência da Computação. In Anais do $5^{\circ}$ Seminário Nacional de Inclusão Digital (SENID). Cruz Alta: UNICRUZ, 02-10.

[9] Aline Diesel, Alda Leila Santos Baldez, and Silvana Neumann Martins. 2017. Os princípios das metodologias ativas de ensino: uma abordagem teórica. In Revista Thema 14, 1 (2017), 268-288.

[10] Shreenath Acharya and MN Gayana. 2021. Enhanced Learning and Improved Productivity of Students' using Project Based Learning Approaches for Programming Courses. In Journal of Engineering Education Transformations 34, (2021), 524-530.

[11] Ronney Moreira Castro and Sean Siqueira. 2019. Técnicas Alternativas de Ensino (Aprendizagem Ativa) para Disciplinas da Computação: Um Mapeamento Sistemático no Contexto Brasil. In Anais do Workshop de Informática na Escola (Vol. 25, No. 1, pp. 1409-1413.

[12] Diego Teixeira Witt, Avanilde Kemczinski, and Luciane Mulazani dos Santos. 2018. Resolução de problemas: Abordagens aplicadas no ensino de computação. In Anais do Computer on the Beach (2018), 731-740.

[13] Kathleen M Koenig. 2020. Personal response systems: Making an informed choice. In Active Learning in College Science Journal. Springer, 123-139.

[14] Jose Moran. 2021. Avanços e desafios na educação híbrida. Educação

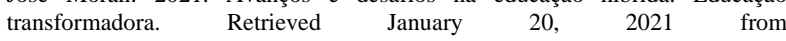
http://www2.eca.usp.br/moran/.

[15] Paul Parsons. 2011. Preparing computer science graduates for the 21st Century. Teaching Innovation Projects 1, 1 (2011).

[16] Jesse Eickholt. 2018. Barriers to active learning for computer science faculty. arXiv preprint arXiv: 1808.02426 (2018)

[17] Joel Michael. 2007. Faculty perceptions about barriers to active learning. In College Teaching 55, 2 (2007), 42-47.

[18] Elisa L Park and Bo Keum Choi. 2014. Transformation of classroom spaces: Traditional versus active learning classroom in colleges. In Higher Education 68, 5 (2014), 749-771.

[19] Williamson Silva, Bruno Gadelha, Igor Steinmacher, and Tayana Conte. 2020. Towards an open repository for teaching software modeling applying active learning strategies. In 2020 IEEE/ACM 42nd International Conference on Software Engineering: Software Engineering Education and Training (ICSESEET), IEEE 162-172.

[20] Alan Hevner and Samir Chatterjee. 2010. Design science research in information systems. In Design research in information systems. Springer, 9-22.

[21] Roel J Wieringa. 2014. Design science methodology for information systems and software engineering. Springer.

[22] Arto Vihavainen, Jonne Airaksinen, and Christopher Watson. 2014. A systematic review of approaches for teaching introductory programming and their influence on success. In Proceedings of the tenth annual conference on International computing education research, 19-26. 